\title{
Spectral phase effects on nonlinear resonant photochemistry of 1,3-cyclohexadiene in solution
}

\author{
E. C. Carroll, B. J. Pearson, A. C. Florean, P. H. Bucksbaum, and Roseanne J. Sension ${ }^{\text {a) }}$ \\ FOCUS Center, University of Michigan, 500 East University Avenue, Ann Arbor, \\ Michigan 48109-1120
}

(Received 14 October 2005; accepted 29 December 2005; published online 17 March 2006)

\begin{abstract}
We have investigated the ring opening of 1,3-cyclohexadiene to form 1,3,5-cis-hexatriene (Z-HT) using optical pulse shaping to enhance multiphoton excitation. A closed-loop learning algorithm was used to search for an optimal spectral phase function, with the effectiveness or fitness of each optical pulse assessed using the UV absorption spectrum. The learning algorithm was able to identify pulses that increased the formation of Z-HT by as much as a factor of 2 and to identify pulse shapes that decreased solvent fragmentation while leaving the formation of Z-HT essentially unaffected. The highest yields of Z-HT did not occur for the highest peak intensity laser pulses. Rather, negative quadratic phase was identified as an important control parameter in the formation of Z-HT. (C) 2006 American Institute of Physics. [DOI: 10.1063/1.2168454]
\end{abstract}

\section{INTRODUCTION}

Control over physical processes in molecules via phase shaping of an ultrafast laser pulse has now been widely demonstrated. ${ }^{1}$ Experiments reported in the literature range from strong-field multiphoton control of molecular rearrangements in the gas phase ${ }^{2}$ to control of excited-state populations in resonant transitions in liquids. ${ }^{3,5}$ These experiments represent important steps toward the ultimate goal: optical control of chemical reactivity. However, coherent control of molecular rearrangement and chemical reactivity in the condensed phase faces several challenges that distinguish it from control in atomic systems or simple control of an excited-state population. These challenges include dissipation, dephasing, and interference by nonlinear interactions of high intensity pulses with solvent molecules. In the face of these complexities, the optical control of solution phase reactivity remains an important active field of research. A significant advance was recently reported on this subject: the control of a solution phase cis-trans photoisomerization following "one-photon" excitation. ${ }^{6}$ In this paper we report on a series of experiments exploring the pulse-shape dependence of the multiphoton photochemistry of 1,3-cyclohexadiene in solution.

1,3-cyclohexadiene (CHD) undergoes an optically induced ring-opening reaction to form 1,3,5-cis-hexatriene (ZHT). This ring opening is analogous to the chromophore dynamics through which 7-dehydrocholesterol forms previtamin $\mathrm{D}$, a reaction that occurs in human skin. ${ }^{7} \mathrm{~A}$ schematic of the CHD ring opening and the absorption spectra of CHD and Z-HT are shown in Fig. 1. Extensive experimental ${ }^{8}$ and theoretical investigations ${ }^{9-14}$ have led to a detailed understanding of the CHD isomerization: Upon excitation in the UV absorption band, the electronic population is transferred from the $1 A$ ground state to the $1 B$ excited state. The wave packet produced in this excitation process accelerates

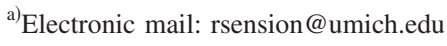

along the torsional reaction coordinate. ${ }^{15}$ Before significant nuclear motion can occur on the $1 B$ surface the wave packet is funneled through a conical intersection onto the $2 A$ excited state where most of the nuclear ring-opening dynamics occur. CHD returns to the ground state through several conical intersections between the $1 A$ and $2 A$ potential-energy surfaces. ${ }^{9,16}$ The internal conversion to the ground electronic state is complete within a few hundred femtoseconds of excitation to the one-photon-allowed electronic state. Sixty percent of the excited molecules relax back to form vibrationally hot $\mathrm{CHD}$, while the remaining $40 \%$ isomerize to form $c \mathrm{Zc}$-HT with cis (or more precisely, gauche) configurations around both of the single bonds. Within 50 ps most of the vibrationally excited Z-HT undergoes conformational relaxation to the stable $t \mathrm{Z} t$-HT form with trans configurations

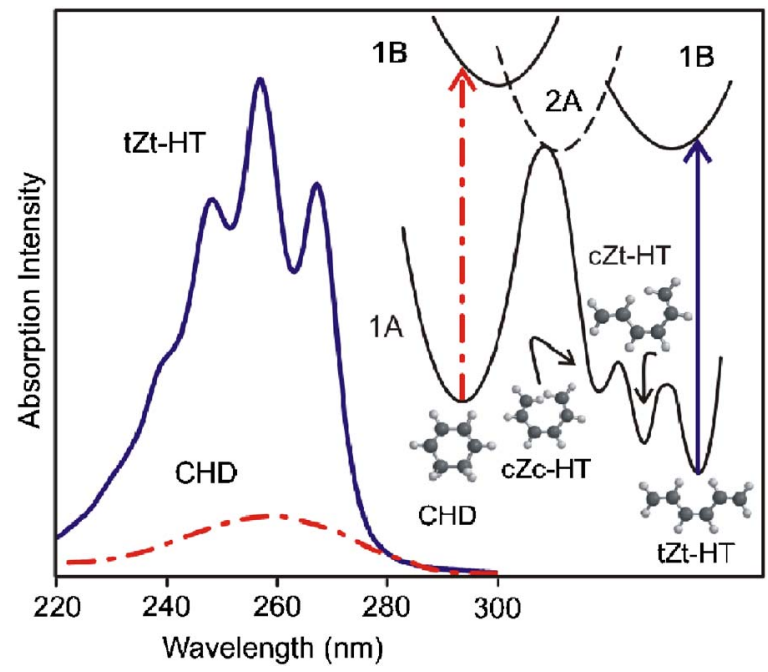

FIG. 1. (Color online) Steady-state absorption spectra of 1,3-cyclohexadiene (CHD, dot-dashed line) and cis-1,3,5-hexatriene (Z-HT, solid line). The oscillator strength of HT is approximately eight times that of CHD (Ref. 6). A cartoon (not to scale) of the electronic states and molecular conformations playing a role in the photochemistry of CHD is also shown in the figure. 
around both of the single bonds, while a few percent is trapped as $c \mathrm{Zt}$ - $\mathrm{HT}$, relaxing to the $t \mathrm{Zt}$-HT ground-state configuration within a nanosecond. ${ }^{17}$

Recently CHD has been recognized as a candidate for coherent control and for potential use as a molecular logic switch. ${ }^{18}$ Direct one-photon $1 A \rightarrow 2 A$ transitions are effectively forbidden. With linear excitation, dynamics on the $2 A$ state must proceed under the momentum of the wave packet shaped on the optically accessible $1 B$ state. Therefore, theoretical investigations of coherent control in $\mathrm{CHD}$ have focused on wave-packet shaping on the time scale of the $1 B$ lifetime, $10-30 \mathrm{fs}^{10,19,20}$ de Vivie-Riedle and co-workers have developed wave-packet propagation simulations showing that the quantum yield of the Z-HT formation depends on the initial condition of the wave packet on the $2 A$ surface. $^{10-13}$

In the $\mathrm{CHD} \rightarrow \mathrm{Z}$-HT reaction out-of-plane torsional motions account for much of the nuclear dynamics in the ringopening process. The primary difference between the equilibrium geometry of CHD and the open-ring structure of $c \mathrm{Zc}$-HT is an increase in the distortion from planarity in the latter molecule. Although the ring-opening reaction occurs on the $2 A$ state, torsional distortion is already active in the $1 B$ state as evidenced in the resonance Raman excitation profile. ${ }^{15}$ The lowest ground-state vibrational modes detected in resonance Raman, at 203 and $507 \mathrm{~cm}^{-1}$, were assigned to $\mathrm{C}=\mathrm{C}$ torsions.

It is reasonable to expect that excited state coherent intramolecular dynamics will influence the CHD ring-opening reaction. Complete active space self-consistent field (CAS$\mathrm{SCF}$ ) and multistate complete active space second order perturbation theory (MS-CASPT2) calculations have identified two conical intersections (CIs) connecting the $S_{1}(2 A)$ and the $S_{0}(1 A)$ potential-energy surfaces. ${ }^{9,16,21}$ Hoffman et al. pointed out that the minimum-energy excited-state configuration corresponding to these two primary $S_{1} / S_{0}$ conical intersections can be reproduced relatively well by six dominant vibrational modes, where the lowest-frequency mode, $196 \mathrm{~cm}^{-1}$, projects strongly onto the torsional $\mathrm{C}=\mathrm{C}$ reaction coordinate. ${ }^{11,14}$ These two conical intersections represent different reaction pathways. The higher-energy CI dominates the ultrafast internal conversion and is consistent with the observed $40 \%$ yield for the Z-HT formation under linear excitation conditions. Hoffman and de Vivie-Riedle, ${ }^{12}$ and Geppert and de Vivie-Riedle ${ }^{19}$ determined that passage through the lower-energy CI is slower and results in lower yield for Z-HT (ca. 30\%). Following internal conversion from the $1 B$ state to the $2 A$ state the reaction proceeds through both pathways. To effect control via excitation to the $1 B$ state, approximately $30000 \mathrm{~cm}^{-1}$ of coherent bandwidth was required in the calculations of Geppert and de Vivie-Riedle. ${ }^{19}$ In this broad bandwidth limit, hexatriene production depends on the shape of the laser pulse. Though these theoretical results demonstrate the potential for coherent control of CHD, the optimal pulse shapes are not easily attained in the laboratory.

Multiphoton excitation of CHD can result in direct excitation of the $2 A$ state, bypassing the limitation of the $1 B$ lifetime. Thus, a significant question is whether coherent

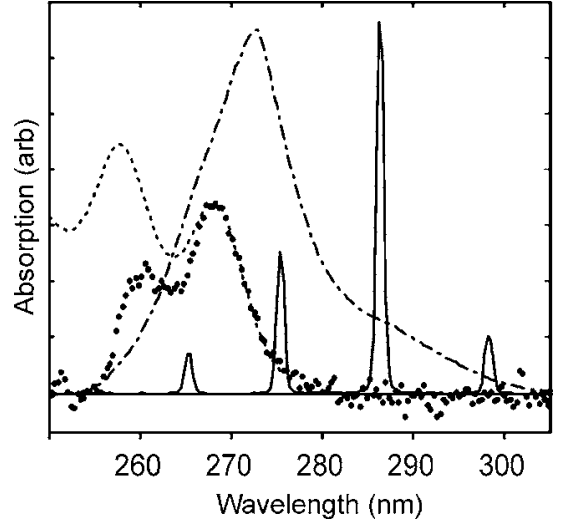

FIG. 2. Comparison of the probe pulses used for detection of the photoproduct Z-HT. The anticipated difference spectrum for the production of Z-HT from CHD in cyclohexane (dashed line) is shown for comparison. The discrete probe using the UV lines generated in a high-pressure $\mathrm{CO}_{2}$ Raman cell consists of narrow peaks at 266, 276, 287, 299, and $312 \mathrm{~nm}$. The dot-dashed line illustrates a typical UV continuum probe. The points represent a difference spectrum obtained using the UV continuum probe and a transformlimited pump pulse $(\lambda=268 \mathrm{~nm})$. This spectrum shows resolution of the full 0-0 peak of Z-HT.

control of the CHD to Z-HT photoisomerization is possible in the case of multiphoton absorption with longer pulses. To study this problem we employed narrower bandwidth pulses, 125 and $310 \mathrm{~cm}^{-1}$, with a central wavelength of $800 \mathrm{~nm}$ $\left(1.25 \times 10^{4} \mathrm{~cm}^{-1}\right)$. The transform limits of these pulses are ca. 120 and $50 \mathrm{fs}$, respectively. Resonant excitation of the $\pi \pi^{*}$ transition requires simultaneous absorption of three photons. The limited bandwidth available forbids impulsive excitation of all but the lowest-frequency modes of CHD. Nonetheless we have found that spectral phase affects the production of Z-HT through multiphoton excitation, even when the pulse features are on the time scale of the ring opening. Negative chirp is identified as an important control parameter in the formation of Z-HT.

\section{EXPERIMENT}

Experiments were conducted independently on two different Ti:sapphire laser systems. Both laser systems produced amplified pulses with a maximum energy of $1 \mathrm{~mJ}$ and a central wavelength of $800 \mathrm{~nm}$. One laser system operates at a repetition rate of $10 \mathrm{~Hz}$ and has $8 \mathrm{~nm}\left(125 \mathrm{~cm}^{-1}\right)$ full width at half maximum (FWHM) of spectral bandwidth. The second laser system operates at $1 \mathrm{kHz}$ and has a bandwidth of $20 \mathrm{~nm}$ FWHM $\left(310 \mathrm{~cm}^{-1}\right)$. All experiments were conducted in a pump-probe configuration with a probe pulse at a fixed time delay, nanoseconds after the shaped pump pulse. Thus, the probe detects the formation of the permanent Z-HT product long after relaxation to the stable $t \mathrm{Zt}$-HT conformer. Differential absorption of the probe pulse is a direct measure of the concentration of Z-HT created by the pump pulse.

Two types of optical probes were used in these experiments (Fig. 2). For the experiments carried out using the narrower bandwidth laser, the fourth harmonic of a Nd:YAG (yttrium aluminum garnet) laser was focused into a $1 \mathrm{~m}$ cell containing $\mathrm{CO}_{2}$ at high pressure. Stimulated Raman scatter- 
ing in the $\mathrm{CO}_{2}$ gas produced five narrow lines in the ultraviolet (UV). The $10 \mathrm{~ns}$ probe pulses were centered at a time delay of $20 \mathrm{~ns}$ after the pump pulse.

Experiments conducted with the $1 \mathrm{kHz}$ laser system with a $20 \mathrm{~nm}$ bandwidth used a femtosecond ultraviolet continuum probe. The compressed fundamental of the laser was focused into a $1 \mathrm{~cm}$ cell of ethylene glycol where self-phase modulation (SPM) and other nonlinear effects ${ }^{22}$ broadened the spectrum into a white light continuum. The white light was collimated and focused by a fast achromatic lens ( $f$ $=30 \mathrm{~mm}$ ) into a beta barium borate $(\mathrm{BBO})$ crystal for type-I doubling. Typically, a bandwidth of $60-80 \mathrm{~nm}$ of visible radiation was adequately phase matched for second-harmonic generation (SHG) producing a continuum tunable from $\sim 260$ to $320 \mathrm{~nm}$. The probe pulses were delayed to arrive at the sample either 1 or $12 \mathrm{~ns}$ after the pump pulse. Compensation for temporal dispersion in the probe pulse was unnecessary because the dispersion was insignificant compared with the nanosecond delay between the pump and probe pulses in these experiments.

Control of the spectral phase of the $800 \mathrm{~nm}$ laser pulse was realized with a pulse shaping system based on a zerodispersion Fourier filter design using an acousto-optic modulator (AOM) as introduced by Warren et $a l^{23}$ The shaped pulse was amplified further in a two-to-five-pass Ti:sapphire amplifier and compressed using a grating pair. $^{24}$

Accurate optimal control theory calculations of the CHD system in solution are challenging research problems in their own right, stretching present capabilities. Instead, full timedependent solutions for laser-induced photoisomerization can be found by experimental searches using a learning algorithm in conjunction with a closed feedback loop. ${ }^{25}$ The learning algorithm used in these experiments is a modified genetic algorithm (GA) and is described in detail elsewhere. ${ }^{24}$

For feedback into the learning algorithm, the optical probe transmitted through the CHD sample was collected with a multichannel spectrometer (Ocean Optics S2000). An optical chopper was used to block the pump pulse to alternately obtain a pumped photoproduct spectrum $\left(I_{\text {sig }}\right)$ or reference spectrum $\left(I_{\text {ref }}\right)$ for the transmitted probe. From each pair of spectra a difference spectrum was calculated as $\Delta A(\lambda)=-\log \left[I_{\text {sig }}(\lambda) / I_{\text {ref }}(\lambda)\right]$

Two control goals were used. The first control goal (goal 1) monitored Z-HT production defined in terms of the objective functional $J(E)$,

$$
J(E)=\int_{260}^{280} \Delta A(\lambda, E) d \lambda
$$

where $\lambda$ is the probe wavelength and $E$ is the applied electric field of the optical pulse. Because both of the probes used in these experiments sample the regions of the spectrum inside and outside of the expected hexatriene absorption, the second control goal was designed with a penalty for absorption outside of the region of the Z-HT spectrum,

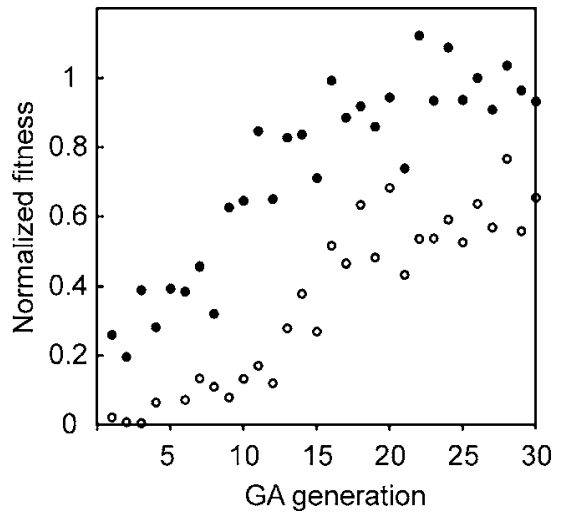

FIG. 3. Trajectory illustrating the fitness of the best pulse for each generation (filled) and median fitness of each generation (open circles) of a typical experiment. Fitness is normalized to the plateau rather than absolute best fitness.

$$
C(E)=\int_{280}^{300} \Delta A(\lambda, E) d \lambda
$$

Goal 2, defined as $J(E)-C(E)$, permits some discrimination between signals corresponding to the formation of Z-HT and signals corresponding to the background effects in the solvent.

The samples were prepared by diluting 1,3cyclohexadiene (Aldrich) with spectroscopic grade cyclohexane or hexane to a concentration of $4 \mathrm{mM}$. The sample was flowed though a 1-mm-thick quartz flow cell at a rate sufficient to replenish the sample between laser pulses. Immediately following the search algorithm, a fresh sample of the solution was irradiated with pulses having the highest fitness pulse shape. Steady-state spectra for the samples were obtained using a Shimadzu UV-1601 UV-VIS spectrometer.

\section{RESULTS}

\section{A. Feedback control}

Genetic algorithm searches were successful in increasing the transient absorption signature for Z-HT. Pulse fitness improved by as much as an order of magnitude or more between the first and last generations of the GA (Fig. 3). Among the set of GA solutions, certain common attributes were robust under variations in pulse energy and bandwidth. Notably, in all of these experiments the pulse that produced the strongest transient absorption in the CHD solution was not transform limited despite the fact that excitation was a multiphoton process.

Difference spectra from a typical GA run using the Raman probe are summarized in Fig. 4. The pulse found to best satisfy control goal 2 results in the absorption of the 266 and $276 \mathrm{~nm}$ lines more than a factor of 2 stronger than the transform-limited pulse. The transmission of the 287, 299, and $312 \mathrm{~nm}$ lines was not significantly affected. A second run with a higher pulse energy reproduced these results and recovered a nearly identical optimal pulse shape. These experiments demonstrated a clear ability of the GA to generate pulse shapes resulting in optimized differential photoproduct 


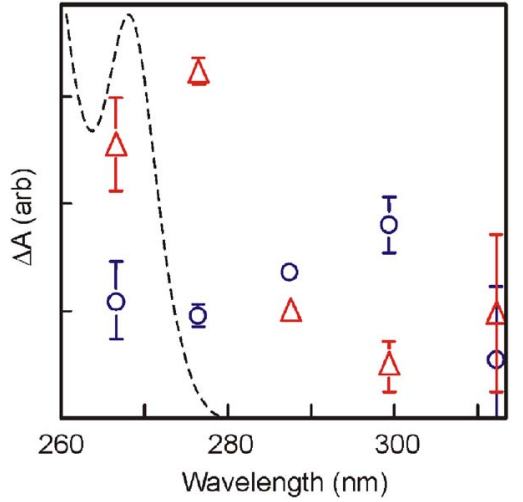

FIG. 4. (Color online) Sample difference spectra that best met the control goal in GA searches using the $\mathrm{CO}_{2}$ Raman probe. The dashed line is the expected difference spectrum for the production of cis-1,3,5-hexatriene from CHD. The pulse optimized under control goal 2 produced strong absorption for two of the probe lines (triangles) compared with the transform-limited pulse (circles).

absorption in solution. However, the sparse probe spectrum is insufficient to uniquely identify the products contributing to the difference spectrum.

Peak structure in the difference spectrum corresponding to Z-HT becomes clear when the UV continuum probe pulse is used. Data obtained during one series of experiments are plotted in Fig. 5. Randomly generated pulse shapes typically produced weak, featureless absorption extending over all probe wavelengths. This broad background is not part of the expected Z-HT absorption spectrum (see Fig. 2), but was observed in all multiphoton experiments.

Excitation of a solvent-only sample with a transformlimited pulse results in an enhanced background signal. The solvent-only difference spectrum is slowly varying over this spectral region and does not exhibit the characteristic 0-0 transition of Z-HT. Excitation of CHD in hexane results in a

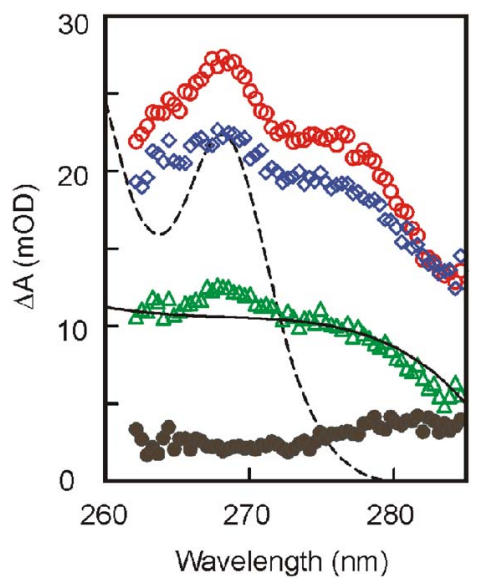

FIG. 5. (Color online) Difference spectra observed following excitation of $\mathrm{CHD}$ in hexane with an $800 \mathrm{~nm}$ pulse. The time delay of the probe was $1 \mathrm{~ns}$. Dashed line: the difference between the steady-state spectra of Z-HT and CHD. Solid line: fit to the difference spectra observed following excitation of solvent-only samples with transform-limited pulses. Filled circles: the difference spectrum produced by excitation of CHD in hexane with randomly generated pulse shapes. Diamonds: the difference spectrum observed following excitation of CHD in hexane with transform-limited pulses. Open circles: the difference spectrum obtained by the GA with the control goal 1 . Triangles: the difference spectrum obtained by the GA with control goal 2 .

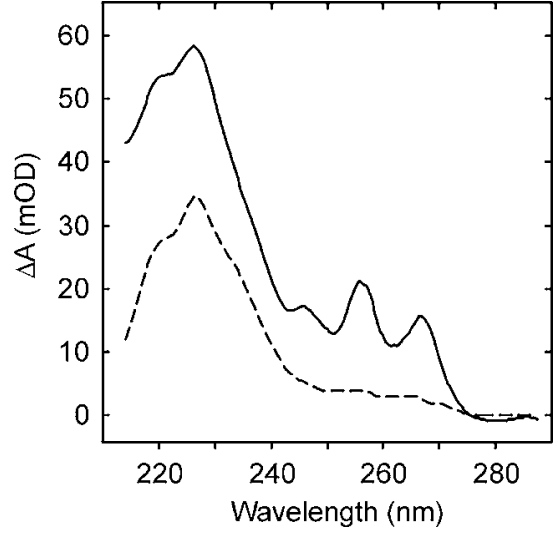

FIG. 6. UV-VIS spectra obtained from irradiated samples of the CHD solution (solid) and neat hexane (dashed). The $800 \mathrm{~nm}$ pulse is transform limited. The structure of the hexatriene spectrum is clearly resolved following excitation of CHD. Additional photoproducts are seen in both the CHD solution and pure hexane. However, Z-HT is observed only in the CHD solution.

difference spectrum with a broad background, similar to that observed in solvent only, with the addition of a distinct absorption peak characteristic of the 0-0 transition of Z-HT. Analysis of the optimized difference spectra requires fitting the data to a linear combination of the background spectrum obtained from the solvent-only scans and the known Z-HTCHD difference spectrum. The spectral structure of the solvent transient is assumed to be invariant with pulse shape for pulses with high fitness.

The GA algorithm with control goal 1 results in a modest increase in the background $(\sim 13 \%)$, but a significant increase in the 0-0 transition of Z-HT ( 100\%) (see Fig. 5). Control goal 2 was designed to suppress the absorption background or favor pulse shapes that discriminated between absorption regions. For the data plotted in Fig. 5, the GA successfully suppressed the background $(\sim 50 \%$ reduction $)$ while leaving the signal due to the $0-0$ transition of Z-HT essentially the same as for the transform-limited pulse $(\sim 5 \%$ increase). In a second set of experiments comparing control goals 1 and 2, data not shown, the optimization resulted in a $33 \%$ suppression of background for goal 2 over goal 1 at the expense of a corresponding decrease in the Z-HT signal. For all of the experiments, control goal 1 was effective in increasing the 0-0 Z-HT peak, often with a significant concomitant increase in the background signal. Control goal 2 reduced solvent background processes, sometimes at the expense of the Z-HT signal. The definition of the search goal clearly influenced the solutions found by the GA.

\section{B. Steady-state spectroscopy}

Steady-state UV absorption spectroscopy was used to analyze photoproducts produced by multiphoton excitation of the solvent and to confirm the enhanced production of Z-HT by the optimized laser pulses. Although absorption to electronic states of the solvent is a higher-order process than excitation of CHD (requiring at least four or five photons), the broad backgrounds observed in Figs. 4 and 5 demonstrate the presence of additional photoproducts formed in these solvents. To clarify the solvent photochemistry the samples of 


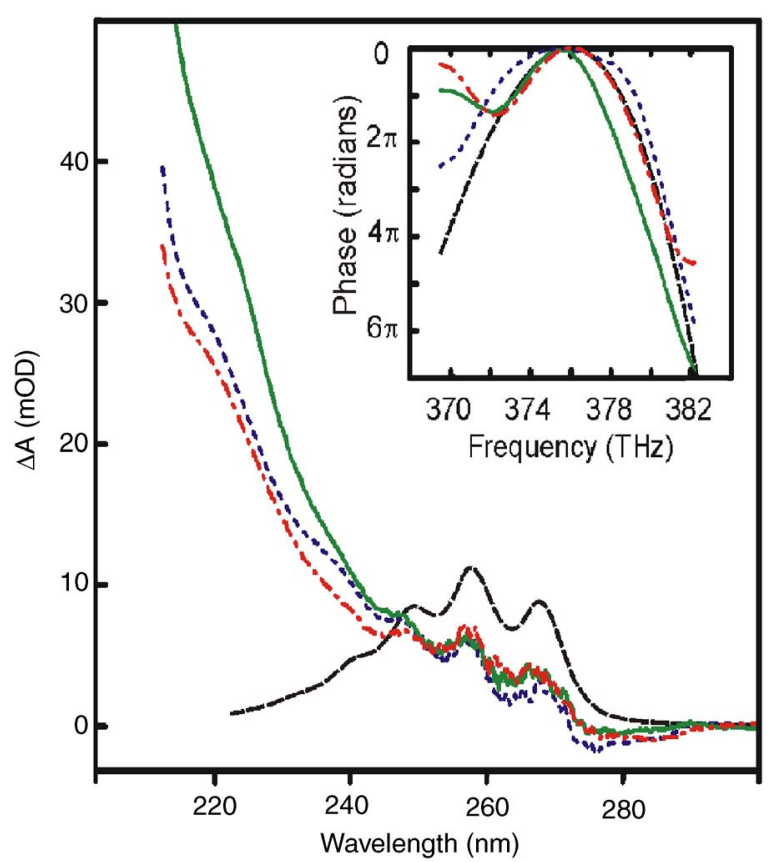

FIG. 7. (Color online) UV-VIS difference spectra were obtained as the difference between a sample irradiated with the optimal pulse and a reference sample irradiated with a nearly transform-limited pulse. Difference spectra for three pairs of pulse shapes are shown (solid, small dashes, and dot-dashed lines). A reference spectrum for Z-HT is also shown (large dashes). The inset shows the phase $\Delta \phi(\omega)$ responsible for the corresponding difference spectra. Negative chirp is a common feature among the pulses with higher Z-HT yield. A phase function with pure linear chirp, $\phi^{\prime \prime}(\omega)=$ $-2.0 \times 10^{4}$, is shown in the inset for reference (large dashes).

pure solvent and the samples of CHD solution were irradiated under identical experimental conditions. A similar deep UV background was observed in both the CHD solution and in pure hexane (Fig. 6). Photoproducts can be difficult to identify by linear spectroscopy due to the broad and relatively featureless UV absorption spectra of most small alkanes, alkenes, and alkynes. However, the peaks at 221 and $227 \mathrm{~nm}$ are consistent with the formation of hex-3-en-1-yne $\left(\mathrm{C}_{6} \mathrm{H}_{8}\right)$ and possibly 1,3-pentadiene. ${ }^{26}$ In general, the deep UV background suggests complex fragmentation and rearrangement of the solvent. These higher-order processes are probably in competition with the formation of, and certainly complicated detection of, the desired photoproduct, Z-HT. To some extent, pulse shapes favoring Z-HT production also favor solvent excitation.

On the other hand, Z-HT was detected only in the CHD solution. We can therefore conclude that Z-HT is produced from CHD rather than fragmentation or rearrangement of the solvent. As an aside, the experiments described above were carried out in hexane rather than cyclohexane because irradiation of neat cyclohexane resulted in small yields of HT in the absence of CHD.

Steady-state UV-VIS spectra of the samples irradiated with the optimal pulse shapes were acquired to assess the effectiveness of the pulses found by the GA. Difference spectra were obtained comparing the CHD samples irradiated with either by the optimal pulse shape determined by the GA or by a reference pulse shape that was close to transform limited. Difference spectra for three independent runs are shown in Fig. 7. The difference in phase for each pair, $\Delta \phi(\omega)=\phi(\omega)_{\mathrm{GA}}-\phi(\omega)_{\text {ref }}$, is responsible for a positive net yield in Z-HT. Because the reference pulse in each pair is nearly transform limited $\left[\Delta \phi(\omega) \approx \phi(\omega)_{\mathrm{GA}}\right]$, we can conclude that the difference in phase represents an important control parameter in the multiphoton production of Z-HT. Negative chirp is a common feature among the pulses producing a higher Z-HT yield. The relatively low intensity of the optimal pulse suggests that multiphoton absorption is in competition with the mechanisms of interest in the ring opening. A more detailed analysis of the optimized pulse shapes is considered in the Discussion section below.

\section{DISCUSSION}

The data described above illustrate the production of Z-HT from CHD through interaction with $800 \mathrm{~nm}$ pulses. Because these experiments rely on multiphoton absorption to initiate the CHD ring opening, we can expect that the transient absorption will depend strongly on pulse intensity $I$. The probability for three-photon absorption scales is $I^{3}$. Therefore we expected to find that the optimal pulse had the highest peak intensity, i.e., a Fourier-transform-limited pulse. This was not found. Instead, the production of Z-HT is optimized for specific non-transform-limited pulses. Clearly, high peak power is not the only important criterion for the production of Z-HT in the CHD solution.

Conclusions need not be drawn from only the limited number of optimal pulses. While the optimal pulses found in these experiments exhibit several obvious common features, each GA search tests several hundred different pulse shapes. Phase and amplitude information were saved for every pulse shape tested. As a result the GA search set comprises a data set much larger than the set of optimal pulse shapes. Important insights may arise from a systematic analysis of correlations between pulse characteristics and pulse performance for all of the pulses in this search set. ${ }^{27}$ Duplicate pulse shapes, tested multiple times (clones), have been removed from the data sets and are represented by an average fitness.

\section{A. Comparison of pulse fitness and peak power}

The influence of peak power can be estimated by calculating the nonlinear power spectrum of each pulse. ${ }^{28}$ The $n$ th-order nonlinear power spectrum is proportional to an $n$th order convolution of fields where the complex field of the fundamental pulse is given by $E(\omega)=A(\omega) \exp [i \phi(\omega)]$, where $A(\omega)$ and $\phi(\omega)$ represent the amplitude and phase programmed onto the AOM.

Several multiphoton experiments in the literature compensate for the intrinsic intensity dependence by taking the fitness as a ratio between two nonlinear processes of the same order. ${ }^{5,29,30}$ The common dependence of the nonlinear processes on the integrated nonlinear power spectrum, $\int S_{n}(\omega) d \omega$ cancels in the ratio, leaving the fitness function dependent only on the properties of the molecular system under study. This method has proved useful in isolating small pulse-shape dependencies, in experiments which would otherwise have discovered only the trivial result of a transform- 


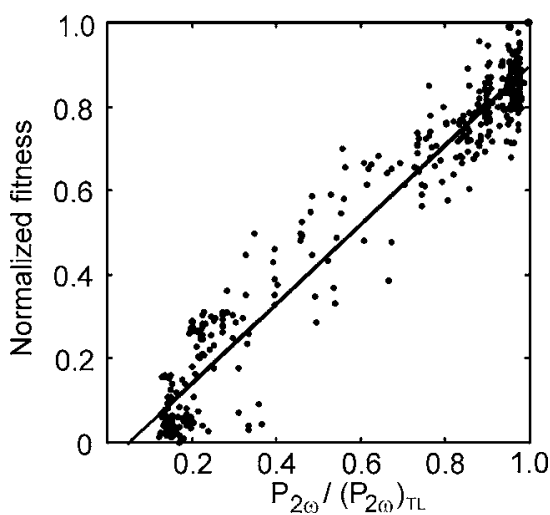

FIG. 8. Correlation between the pulse fitness and the calculated nonlinear power for a GA experiment with the goal of doubling $800 \mathrm{~nm}$ pulses in a BBO crystal. The nonlinear power spectrum was calculated using calibrated phase and amplitude profiles for every pulse tested in the algorithm. Both the measured and calculated powers have been normalized to the pulse with the highest conversion efficiency. The measured second-harmonic power is highly correlated $(r=0.97)$ with the calculated $P_{2 \omega}$.

limited pulse. In the present experiments, effective, nontrivial pulses were found despite the intensity dependence.

The intrinsic pulse intensity can easily be calculated from the known values of $A(\omega)$ and $\phi(\omega)$ and compared with the observed fitness. An application of this analysis to a GA search on second-harmonic generation is illustrated in Fig. 8. A 1-mm-thick BBO crystal was placed at the position of the CHD sample. Second-harmonic power was measured by a photodiode and was fed back to the learning algorithm. Assuming that second-harmonic efficiency is independent of wavelength over the bandwidth of the laser, the secondharmonic signal should be proportional to the integrated nonlinear power spectrum $P_{2 \omega}=\int S_{2}(\omega) d \omega$, where

$$
S_{2}(\omega)=\left|\int_{-\infty}^{\infty} d \Omega A(\Omega) A(\omega-\Omega) e^{i[\phi(\Omega)+\phi(\omega-\Omega)]}\right|^{2}
$$

is the spectral density of the square of the shaped electromagnetic field.

The measurements and the calculated nonlinear integrated power show strong correlation (Fig. 8). Deviations from a line of unit slope reflect collective errors due to propagation of the shaped pulse, saturation effects in the post-AOM amplification, imperfect calibration of the pulse shaper, and, to a lesser effect, imperfect alignment of the doubling crystal. Taking into account all sources of experimental noise, we can be confident that the pulse at the sample has peak power within $20 \%$ of the calibrated value. The pulse shape with the highest conversion efficiency was used to calibrate the phase of the AOM in the CHD experiments described in the next paragraph.

A similar analysis is now applied to the GA searches for optimal Z-HT formation from CHD. Because at least three photons are required for excitation of $\mathrm{CHD}$ to the lowest excited electronic states, pulse fitness is plotted with respect to the integrated third-order nonlinear power $P_{3 \omega}$ $=\int S_{3}(\omega) d \omega$. The effective third-order power spectrum is
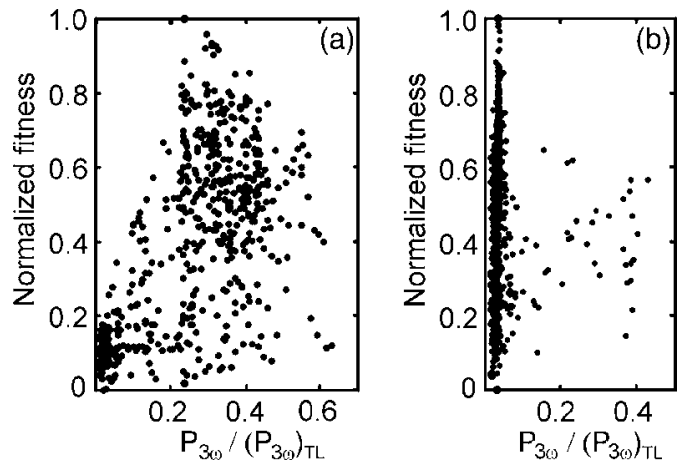

FIG. 9. Correlation between the pulse fitness and calculated nonlinear power for two different GA experiments using control goal 1. The nonlinear power spectrum was calculated using calibrated phase and amplitude profiles for every pulse tested in the algorithm. The GA experiment in (a) had the probe at a relatively short delay of ca. $1 \mathrm{~ns}$, while the GA experiment in (b) had the probe at a long time delay of ca. $12 \mathrm{~ns}$.

$$
\begin{aligned}
S_{3}(\omega)= & \mid \iint d \Omega_{1} d \Omega_{2} A\left(\Omega_{1}\right) A\left(\Omega_{2}\right) A\left(\omega-\Omega_{1}-\Omega_{2}\right) \\
& \times\left. e^{i\left[\varphi\left(\Omega_{1}\right)+\varphi\left(\Omega_{2}\right)+\varphi\left(\omega-\Omega_{1}-\Omega_{2}\right)-\varphi_{\mathrm{TL}}\right]}\right|^{2} .
\end{aligned}
$$

The results from two GA searches are summarized in Fig. 9. These two searches were conducted with similar pulse energy, pulse bandwidth, and the same experimental goal. They differed in the time delay between the pump and probe pulses. Figure 9(a), with a time delay of 1 ns, showed a clear but weak correlation between pulse fitness and $P_{3 \omega}$. When the time delay is extended to $12 \mathrm{~ns}$ in Fig. 9(b), this correlation is no longer present. These trends are easily distinguished above the experimental noise.

Since the Z-HT product absorption is not influenced by the delay of the probe, we conclude that the correlation with third-order power is dominated by the interaction of the pulse with the solvent, which produces a transient background absorption in the spectral region of the target due to the solvent fragmentation and rearrangement processes described above. When the probe is delayed by $1 \mathrm{~ns}$ the solvent background between 260 and $300 \mathrm{~nm}$ is of more significance than at the longer $12 \mathrm{~ns}$ time delay. No solvent background is observed in this wavelength region in the steady-state measurements plotted in Fig. 6, indicating that the absorption arises from intermediate photoproducts that relax or undergo further reaction and fragmentation on a time scale longer than $1 \mathrm{~ns}$.

\section{B. Comparison of pulse fitness and pulse shape}

It is apparent from the experimental data and analysis that high peak power is not the only important criterion for the production of Z-HT in the CHD solution. Specific pulse characteristics, such as spectral phase, must also be important. Typical high-performance pulse shapes are illustrated in Fig. 10. The most successful pulse shapes in these experiments tend to have negative quadratic phase (linear chirp). This was observed in the comparison of pulses summarized in Fig. 7 and is also evident in the Wigner representation plots of optimal pulses shown in Fig. 10. 

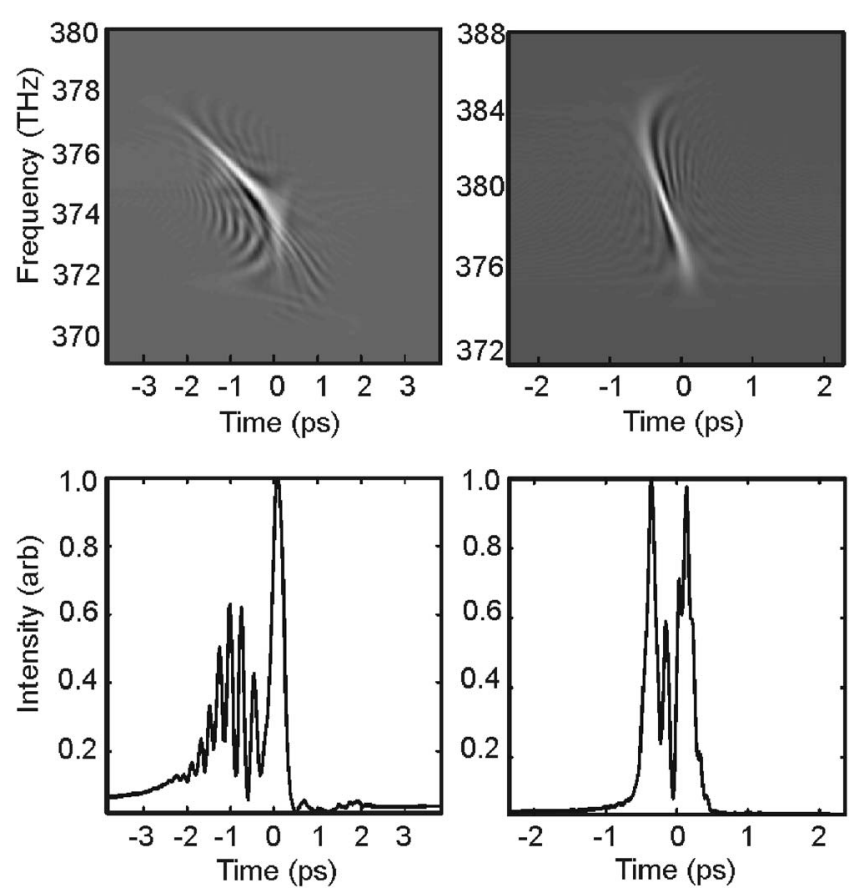

FIG. 10. Wigner representation plots of optimal pulses from a narrow bandwidth experiment (top left) and a broad bandwidth experiment (top right). Each pulse exhibits a negative quadratic phase with $\phi^{\prime \prime}(\omega)=-5.6 \times 10^{4} \mathrm{fs}^{2}$ (left) and $\phi^{\prime \prime}(\omega)=-2.2 \times 10^{4} \mathrm{fs}^{2}$ (right). Negative cubic phase is responsible for the prepulse train evident in the intensity profiles for the two pulses (bottom).

To analyze the role of chirp in more detail, we consider a Taylor-series expansion in the spectral phase,

$$
\begin{aligned}
\phi(\omega)= & \phi\left(\omega_{0}\right)+\phi^{\prime}\left(\omega_{0}\right)\left(\omega-\omega_{0}\right)+\frac{1}{2 !} \phi^{\prime \prime}\left(\omega_{0}\right)\left(\omega-\omega_{0}\right)^{2} \\
& +\frac{1}{3 !} \phi^{\prime \prime \prime}\left(\omega_{0}\right)\left(\omega-\omega_{0}\right)^{3}+\cdots
\end{aligned}
$$

The terms of interest are those representing quadratic and cubic phases, represented by the linear chirp parameter $\phi^{\prime \prime}\left(\omega_{0}\right)$ and the quadratic chirp parameter $\phi^{\prime \prime \prime}\left(\omega_{0}\right)$. We can

TABLE I. Chirp parameters for best pulse shapes. Unless otherwise specified the delay time between the pump and probe is at least $10 \mathrm{~ns}$ and the solvent was hexane. Pulse energies increase moving down the table. All pulse energies are within a factor of 2 .

\begin{tabular}{ccccc}
\hline \hline $\begin{array}{c}\text { Control } \\
\text { goal }\end{array}$ & $\phi^{\prime \prime}(\omega)\left(\mathrm{fs}^{2}\right)$ & $\sigma_{\mathrm{SD}}{ }^{\mathrm{a}}$ & $\phi^{\prime \prime \prime}(\omega)\left(\mathrm{fs}^{3}\right)$ & $\sigma_{\mathrm{SD}}{ }^{\mathrm{a}}$ \\
\hline $2^{\mathrm{b}, \mathrm{c}}$ & $-5.6 \times 10^{4}$ & $4 \times 10^{3}$ & $-2.9 \times 10^{6}$ & $1.9 \times 10^{6}$ \\
$1^{\mathrm{c}}$ & $-2.4 \times 10^{4}$ & $9 \times 10^{3}$ & $2.8 \times 10^{5}$ & $6.4 \times 10^{5}$ \\
$2^{\mathrm{b}, \mathrm{c}}$ & $-6.9 \times 10^{3}$ & $3.0 \times 10^{4}$ & $-3.9 \times 10^{5}$ & $2.2 \times 10^{6}$ \\
$1^{\mathrm{a}}$ & $-8.5 \times 10^{3}$ & $1.9 \times 10^{3}$ & $1.5 \times 10^{5}$ & $2.2 \times 10^{5}$ \\
1 & $-2.0 \times 10^{4}$ & $2.4 \times 10^{3}$ & $-3.2 \times 10^{4}$ & $9.8 \times 10^{4}$ \\
$2^{\mathrm{d}}$ & $-2.2 \times 10^{4}$ & $2.8 \times 10^{3}$ & $-2.7 \times 10^{5}$ & $1.5 \times 10^{5}$ \\
$1^{\mathrm{d}}$ & $-1.6 \times 10^{3}$ & $1.4 \times 10^{3}$ & $-8.1 \times 10^{4}$ & $1.1 \times 10^{4}$ \\
$2^{\mathrm{d}}$ & $5.9 \times 10^{3}$ & $4.5 \times 10^{3}$ & $-9.0 \times 10^{4}$ & $2.0 \times 10^{4}$ \\
\hline \hline
\end{tabular}

${ }^{\mathrm{a}}$ The standard deviation for pulses with normalized fitness $>0.8$. This quantity gives an indication of how well the GA solution represents the entire set of pulses tested.

${ }^{\mathrm{b}}$ Narrow bandwidth, $8 \mathrm{~nm}$, pump pulses.

${ }^{\mathrm{c}}$ Solvent was cyclohexane.

${ }^{\mathrm{d}}$ Delay time between the pump and probe is $1 \mathrm{~ns}$
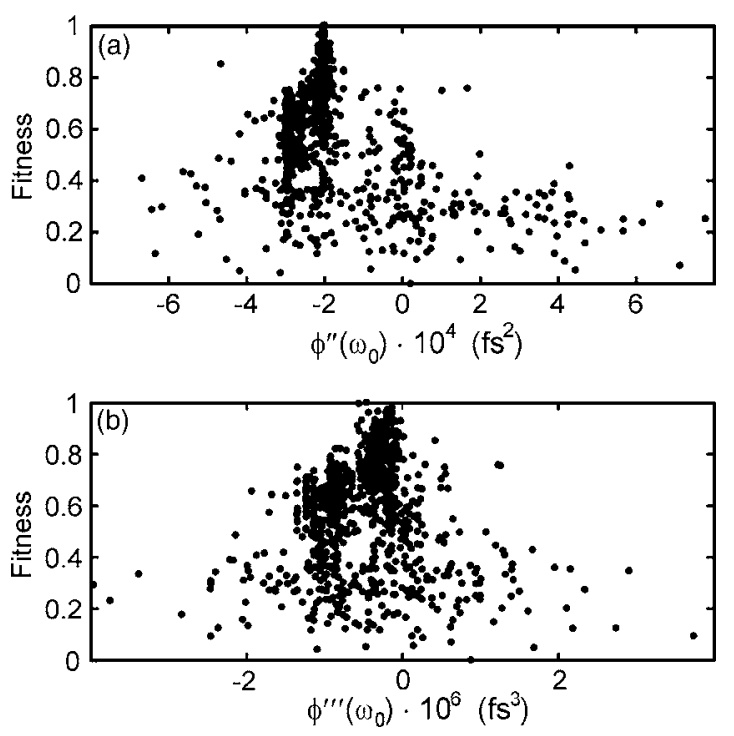

FIG. 11. Pulse performance is compared with the second-order (top) and third-order (bottom) terms from a polynomial fit. Pulse fitness converged to a linear chirp parameter, $\phi^{\prime \prime}\left(\omega_{0}\right)=-2.2 \times 10^{4} \mathrm{fs}^{2}$. Convergence was less clear in the cubic chirp parameter.

ignore the absolute phase $\phi\left(\omega_{0}\right)$ and the group delay incurred by the $\phi^{\prime}\left(\omega_{0}\right)$ term because they are small compared to the long time delay at which the system is probed. A standard least-squares curve-fitting algorithm was used to fit a fourthorder polynomial to the phase of each optimal pulse shape and to all high-fitness pulses. The chirp parameters derived from the polynomial coefficients for the optical pulses analyzed are summarized in Table I.

Analysis of the entire GA data set indicates that highfitness pulses tend to converge to the chirp parameters of the optimal pulse. Such convergence is expected in the space of an important control parameter. The results of this analysis for a typical data set are plotted in Fig. 11. Because there is significant scatter in the data, Table I summarizes both the chirp parameters of the optimal pulse and the variance $\sigma_{\mathrm{SD}}$ in the chirp parameters for pulses with fitness values within $20 \%$ of the best pulse for each GA experiment. It should be noted that the polynomial fit was not well suited to some pulse shapes. The polynomial fit was considered poor if the least-squares value deviated from the minimum value by more than $30 \%$. GA runs where a significant percentage $(>50 \%)$ of high-fitness pulse shapes had unacceptable leastsquares values have been omitted from Table I.

Examination of Table I shows that searches guided by control goal 2 found solutions with large negative cubic phase compared to analogous goal 1 searches. Third-order phase is typically responsible for modulating the pulse intensity at the beat frequency of phase-synchronized optical components. The sign of the chirp $\phi^{\prime \prime \prime}(\omega)$ determines whether the resulting pulse train precedes or succeeds the main pulse. The finding that control goal 2 found optimal pulses with low peak intensity is consistent with the observation that background solvent processes exhibit stronger intensity dependence than the Z-HT formation.

It is worth noting that the search space is limited by a restriction on the algorithm that the change in phase between 
two "pixels" should not exceed $\pi$. The chirp subspace accessible to the pulse shaper has a maximum linear chirp of $\left|\phi^{\prime \prime}(\omega)\right| \approx 8 \times 10^{4} \mathrm{fs}^{2}$, while the maximum quadratic change is $\left|\phi^{\prime \prime \prime}(\omega)\right| \approx 4 \times 10^{6} \mathrm{fs}^{3}$. It is clear that the stochastic sampling of phase space by the GA, as illustrated in Fig. 11, is effectively testing most of the space spanned by the basis vectors $\left[\omega^{2}, \omega^{3}, \omega^{4}\right]$. Although the set of pulse shapes in Fig. 11 does not represent an exhaustive search of chirp space, enough chirps were tested to determine that positive chirp was not a region of high fitness.

\section{Multiphoton control mechanisms in CHD}

Though detailed analysis of both optimal pulse shapes and the correlation between pulse shape and fitness can elucidate optical control parameters, interpretation of the control mechanisms for a complex system remains a challenging task. In the context of nonlinear resonant excitation of a molecular system, phase relationships between components of the optical field can be important in at least three circumstances. In addition, it is possible that the interaction of the intense $800 \mathrm{~nm}$ pulses with the solvent influences the formation of Z-HT indirectly. These four methods of interaction and their application to the $\mathrm{CHD} \rightarrow \mathrm{Z}$-HT ring-opening reaction are discussed below.

\section{Spectral filtering}

The pulse bandwidth may provide multiple $n$-photon pairs to satisfy a single molecular transition. Interference between components of the fundamental field effectively shapes the nonlinear power spectrum governing the dipole response of the molecular system. Meshulach and Silberberg have demonstrated the effect of shaping the nonlinear power spectrum for a narrow-band transition in an atomic vapor. ${ }^{31}$ In a more complex condensed phase system, Lozovoy et al. demonstrated that nonlinear spectral filtering could be used to select excitation in a mixture of dyes with similar onephoton absorption spectra. ${ }^{32}$ In this case, the nonlinear interaction creates a narrow-band nonlinear filter tuned to the spectral region where the two species are most distinguishable.

An important consideration for the experiments on CHD is whether the production of Z-HT is influenced by competition between three-photon excitation to the $1 B$ state and the $2 A$ state. Though the absorption bands for both states are broad, the spectrum $2 A$ state is expected to be redshifted. The nonlinear power spectrum could potentially exploit differences in the absorption spectra of the two multiphoton excitation pathways $1 A \rightarrow 1 B$ or $1 A \rightarrow 2 A$. However, the GA data sets showed little evidence for pressure toward narrow third-order nonlinear spectral features. Therefore, nonlinear spectrum filtering alone is not a dominant mechanism in the optimization of pulse shapes for the production of Z-HT from CHD.

\section{Excited-state population}

Molecular systems often have broad absorption features where the density of states is sufficiently large that the bandwidth of the ultrafast laser will encompass many resonances.
In this situation a shaped optical pulse may satisfy multiple resonances in a molecular system and influence excited-state population and/or wave-packet dynamics. Optical manipulation of excited-state population has been described for several systems. In one-photon resonant experiments probing the emission of dye molecules it is determined that a positive linear chirp enhances fluorescence for intense pump pulses, while negative chirp suppresses fluorescence. ${ }^{33}$ The suppression of excited-state population by negative chirp is commonly attributed to the dumping by the excitation pulse as the frequency sweep of the laser pulse follows the Stokes shift of the dye. ${ }^{4,33,34}$ This process has been described as an intrapulse version of the well-known "pump-dump" model, ${ }^{35}$ where the timing of spectral components within the pulse replaces the explicit time delay of Tannor and Rice.

Recently, Geppert and de Vivie-Riedle ${ }^{19}$ used optimal control theory in a calculation to force the CHD reaction to proceed through the less favorable minimum-energy conical intersection described in the Introduction above. The optimal pulse is negatively chirped, with a very broad spectrum (ca. $\left.3 \times 10^{4} \mathrm{~cm}^{-1}\right)$. They conclude that the frequency sweep of the external field assists stimulated emission. The highfrequency components of the wave packet are dumped back to the CHD ground state, leaving the remaining wave packet without enough momentum along the torsional coordinate to reach the higher-energy $C_{2}$ symmetric conical intersection. Instead, the wave packet relaxes toward the targeted lessefficient minimum-energy conical intersection. These results underscore the role of wave-packet momentum in the CHD isomerization, particularly along the torsional reaction coordinate.

Linear chirp is not a universally effective control parameter, however. In a recent experiment attempting control of a photoisomerization reaction, linear chirp was not an effective control parameter in the formation of the cis-isomer of 3$3^{\prime}$-diethyl-2-2'-thiacyanine iodide following ca. $400 \mathrm{~nm}$ excitation. ${ }^{6}$ Instead, the optimal pulse found for the isomerization of this cyanine molecule was nearly transform limited. In their interpretation of this result Vogt et al. cite manipulation of excited-state population via higher electronic states. An intensity dependence in the resonant multiphoton absorption to higher electronic states may account for the near-transform-limited optimal pulse.

A pump-dump mechanism influencing excited-state population is unlikely to play a significant role in the CHD ring-opening reaction investigated in this paper. All of the interactions for excitation of the CHD reactant are multiphoton, thus a mechanism for dumping population involving the ground and excited states is problematic. A mechanism involving two excited states is also unlikely as there is not a strong excited-state absorption, $S_{1}$ or $S_{2} \rightarrow S_{n}$, at $800 \mathrm{~nm}$ or indeed anywhere in the visible spectrum.

\section{Third-order response of a solution}

In addition to the direct three-photon absorption by the CHD solute, it is also possible that the interaction of the intense $800 \mathrm{~nm}$ pulses with the solvent influences the formation of Z-HT indirectly. Several third-order effects are observed in the experiments described here, most significantly 
SPM. The continuum generated by $800 \mathrm{~nm}$ pulses incident on the sample spans the spectral range from the near infrared to the near ultraviolet and appears the same in solution and in pure solvent. It is reasonable to assume that continuum generation deposits some energy in the solvent and the solute, either through stimulated or impulsive Raman scattering at the difference frequencies of the continuum.

The extra energy deposited in the sample likely contributes to the solvent photochemistry and fragmentation observed in these experiments. One of the photoproducts observed in our measurements on pure solvent is consistent with the formation of hex-3-en-1-yne $\left(\mathrm{C}_{6} \mathrm{H}_{8}\right)$. Production of $\mathrm{C}_{6} \mathrm{H}_{8}$ from hexane $\left(\mathrm{C}_{6} \mathrm{H}_{14}\right)$ would involve a removal of three pairs of hydrogen atoms, perhaps facilitated by stimulated Raman scattering in the solvent. In a gas phase experiment, Muller et al. observed that spectral broadening of the $800 \mathrm{~nm}$ pump pulse increased the degree of fragmentation in the multiphoton ionization of toluene vapor. They reasoned that stimulated Raman scattering deposited extra energy in the vibrational modes, making the molecules more prone to fragmentation. ${ }^{36}$

Though not explicitly observed, third-harmonic generation in the solvent may have also contributed to the CHD reaction. Because pure hexane does not guarantee phase matching, third harmonic generation would not propagate or experience gain over a distance longer than the coherence length, given by $L_{c}=c / \Delta \nu$. However, for a CHD molecule to absorb an UV photon, the UV need only propagate on the order of the distance between solute molecules. In a $4 \mathrm{mM}$ solution, the average distance between CHD molecules is approximately $10 \mathrm{~nm}$, whereas the coherence length is more than $30 \mu \mathrm{m}$ for the bandwidths used in these experiments. Even at low efficiency, third-harmonic generation in the solvent provides a way for the system to produce a more favorable single-photon resonant excitation scheme.

\section{Wave-packet dynamics}

Perhaps the most enticing explanation for the influence of optical pulse shape on a molecular system lies in the control of coherent wave-packet dynamics. In one experiment Brixner et al. ${ }^{5}$ found pulse-shape dependence in the twophoton fluorescence from $\left[\mathrm{Ru}(d p b)_{3}\right]^{2+}$ and the styrene laser dye, DCM, that could not be explained by a nonlinear amplitude filter. Their results point to the importance of coherent intramolecular dynamics in a nonlinear excitation scheme. Wave-packet dynamics can be intrinsically important in a chemical reaction where reaction pathways are often determined on a femtosecond time scale.

Lee et al. also reported an experiment to control fluorescence following two-photon excitation of DCM. ${ }^{30}$ Following excitation, DCM undergoes a cis-trans isomerization about a central $\mathrm{C}=\mathrm{C}$ bond. The stable ground-state conformation is the trans-isomer, while the emission band is attributed to an electronic state associated with the cis-isomer. Thus, the fluorescence yield provides a measure of the efficiency of the cis-trans isomerization. Lee et al. found that negative chirp enhanced the fluorescence signal in DCM. In this study, the efficiency of emission is attributed to the localization or narrowing of the wave packet on the initially excited state. A narrow wave packet is proposed to enhance crossing to the emissive state and minimize nonradiative losses on the initially excited state.

In contrast to the one-photon resonant excitation to the $1 B$ state assumed in the theoretical simulations of the CHD $\rightarrow$ Z-HT reaction, the multiphoton excitation of the experiments described here may access either the $2 A$ or the $1 B$ state. While the bandwidth is limited, impulsive excitation of low-frequency torsional modes on the $2 A$ state is possible, especially with the kilohertz laser system where the pulse bandwidth was $310 \mathrm{~cm}^{-1}$.

In the narrowest bandwidth experiments reported here, significant third-order phase results in a pulse train with a period of approximately 250 fs. This feature was reproducible and observed in several independent GA experiments. Weiner et al. demonstrated that matching the repetition rate of a pulse train to a vibrational frequency is an effective method of mode selectively through multipulse impulsive stimulated Raman scattering, and that the impulse is cumulative. ${ }^{37}$ The pulse trains are slower than lowestfrequency Raman modes of CHD, but the period represents the most complex modulation attainable with the bandwidth $\left(125 \mathrm{~cm}^{-1}\right)$. On the other hand, the pulse train repetition is also close to the duration of the CHD reaction, and may simply represent repumping molecules which have already moved through the excited-state dynamics and returned to the CHD ground state.

The GA experiments carried out with broader optical pulses also found a solution with significant negative chirp, but did not reproduce the extended pulse train obtained with the narrower bandwidth pulses. This may reflect additional pathways accessible to the broader pulses. It is interesting that negative chirp is a common feature of the optimal pulses found in the ring-opening reaction of CHD reported here and the pulses found by Lee et al. to optimize DCM isomerization. ${ }^{30}$ Localization or narrowing of the wave packet on the initially excited state of CHD may enhance absorption and photoproduct formation.

\section{CONCLUSIONS}

We have explored spectral phase effects in the photochemistry of CHD in solution. These experiments demonstrate that it is possible to use optical pulse shaping and three-photon absorption to influence the formation of Z-HT from CHD in solution. Using closed-loop feedback, a learning algorithm was able to identify pulses that increased the formation of Z-HT by as much as a factor of 2 over the transform-limited pulse. The genetic algorithm was also able to identify pulse shapes that decreased solvent fragmentation, while leaving the formation of Z-HT essentially unaffected. The highest yields of Z-HT did not occur for the highest peak intensity laser pulses and did not conform to the $I^{3}$ power law naively expected. Genetic algorithm searches consistently found that negative quadratic phase optimized the Z-HT production.

From these experiments we conclude that extremely broadband coherent pulses do not appear to be required for multiphoton production of Z-HT. This is based on the obser- 
vation that shaped pulses could effectively increase the Z-HT yield even if the pulse durations were considerably longer than the $B$-state lifetime. The nonlinear absorption of $800 \mathrm{~nm}$ light which leads to the Z-HT formation is not dominated by the third-order process required to reach the $B$ state. This might indicate other reaction pathways or the importance of wave-packet manipulation following direct excitation to the $A$ state. Further work is required to sort out these possibilities.

The mechanisms for control of the Z-HT formation, however, are difficult to interpret. The types of coherent control theoretically investigated in CHD are intricately tied to wave-packet shaping. In a real condensed phase system, selfphase modulation and/or third-harmonic generation in the solvent will also play a role. These experiments also highlight the importance of carefully chosen fitness goals to optimize the desired product while minimizing side reactions in the solute and in the solvent.

\section{ACKNOWLEDGMENTS}

The authors wish to thank Michael Orozco for help with the UV-VIS spectroscopy and Aaron Linder who helped with the initial experiments. The authors also thank James White for useful discussions on analyzing learning algorithms. This work is supported by the National Science Foundation through Grant Nos. 9987916 and 0078972, and the FOCUS Center at the University of Michigan.

${ }^{1}$ H. Rabitz, R. de Vivie-Riedle, M. Motzkus, and K. Kompa, Science 288, 824 (2000); M. Shapiro and P. Brumer, Adv. At., Mol., Opt. Phys. 42, 287 (2000); S. A. Rice and S. P. Shah, Phys. Chem. Chem. Phys. 4, 1683 (2002); M. Shapiro and P. Brumer, Rep. Prog. Phys. 66, 859 (2003).

${ }^{2}$ A. Assion, T. Baumert, M. Bergt, T. Brixner, B. Kiefer, V. Seyfried, M. Strehle, and G. Gerber, Science 282, 919 (1998); M. Bergt, T. Brixner, B. Kiefer, M. Strehle, and G. Gerber, J. Phys. Chem. A 103, 10381 (1999); R. J. Levis, G. M. Menkir, and H. Rabitz, Science 292, 709 (2001); N. H. Damrauer, C. Dietl, G. Krampert, S. H. Lee, K. H. Jung, and G. Gerber, Eur. Phys. J. D 20, 71 (2002); M. Bergt, T. Brixner, C. Dietl, B. Kiefer, and G. Gerber, J. Organomet. Chem. 661, 199 (2002); R. J. Levis and H. A. Rabitz, J. Phys. Chem. A 106, 6427 (2002).

${ }^{3}$ C. J. Bardeen, Q. Wang, and C. V. Shank, Phys. Rev. Lett. 75, 3410 (1995); J. L. Herek, W. Wohlleben, R. J. Cogdell, D. Zeidler, and M. Motzkus, Nature (London) 417, 533 (2002).

${ }^{4}$ C. J. Bardeen, V. V. Yakovlev, K. R. Wilson, S. D. Carpenter, P. M. Weber, and W. S. Warren, Chem. Phys. Lett. 580, 151 (1997); C. J. Bardeen, V. V. Yakovlev, J. A. Squier, and K. R. Wilson, J. Am. Chem. Soc. 120, 13023 (1998).

${ }^{5}$ T. Brixner, N. H. Damrauer, P. Niklaus, and G. Gerber, Nature (London) 414, 57 (2001).

${ }^{6}$ G. Vogt, G. Krampert, P. Niklaus, P. Nuernberger, and G. Gerber, Phys. Rev. Lett. 94, 068305 (2005).

${ }^{7}$ W. Fuss and S. Lochbrunner, J. Photochem. Photobiol., A 105, 159 (1997); N. A. Anderson, J. J. Shiang, and R. J. Sension, J. Phys. Chem. A 103, 10730 (1999); N. A. Anderson and R. J. Sension, in Liquid Dynamics: Experiment, Simulation, and Theory, ACS Symposium Series 820, edited by J. T. Fourkas (American Chemical Society, Washington, DC, 2002), p. 148.
${ }^{8}$ S. Pullen, L. A. Walker II, B. Donovan, and R. J. Sension, Chem. Phys. Lett. 242, 415 (1995); S. H. Pullen, N. A. Anderson, L. A. Walker II, and R. J. Sension, J. Chem. Phys. 108, 556 (1998); S. Lochbrunner, W. Fuss, W. E. Schmid, and K.-L. Kompa, J. Phys. Chem. A 102, 9334 (1998); N. A. Anderson, S. H. Pullen, L. A. Walker II, J. J. Shiang, and R. J. Sension, J. Chem. Phys. 102, 10588 (1998); N. A. Anderson, C. G. Durfee III, M. M. Murnane, H. C. Kapteyn, and R. J. Sension, Chem. Phys. Lett. 323, 365 (2000).

${ }^{9}$ M. Garavelli, C. S. Page, P. Celani, M. Olivucci, W. E. Schmid, S. A. Trushin, and W. Fuß, J. Phys. Chem. A 105, 4458 (2001).

${ }^{10}$ R. de Vivie-Riedle, L. Kurtz, and A. Hofmann, Pure Appl. Chem. 73, 525 (2001).

${ }^{11}$ A. Hofmann and R. de Vivie-Riedle, J. Chem. Phys. 112, 5054 (2000).

${ }^{12}$ A. Hofmann and R. de Vivie-Riedle, Chem. Phys. Lett. 346, 299 (2001).

${ }^{13}$ A. Hofmann, L. Kurtz, and R. de Vivie-Riedle, Appl. Phys. B: Lasers Opt. 71, 391 (2000).

${ }^{14}$ L. Kurtz, A. Hofmann, and R. de Vivie-Riedle, J. Chem. Phys. 114, 6151 (2001).

${ }^{15}$ M. O. Trulson, G. D. Dollinger, and R. A. Mathies, J. Chem. Phys. 90, 4274 (1989).

${ }^{16}$ P. Celani, F. Bernardi, M. A. Robb, and M. Olivucci, J. Phys. Chem. 100, 19364 (1996); P. Celani, S. Ottani, M. Olivucci, F. Bernardi, and M. A. Robb, J. Am. Chem. Soc. 116, 10141 (1994).

${ }^{17}$ S. H. Pullen, N. A. Anderson, L. A. Walker II, and R. J. Sension, J. Chem. Phys. 107, 4985 (1997).

${ }^{18}$ K.-L. Kompa and R. D. Levine, Proc. Natl. Acad. Sci. U.S.A. 98, 414 (2001).

${ }^{19}$ D. Geppert and R. de Vivie-Riedle, Chem. Phys. Lett. 404, 289 (2005).

${ }^{20}$ J. Manz, K. Sundermann, and R. de Vivie-Riedle, Chem. Phys. Lett. 290, 415 (1998).

${ }^{21}$ M. Garavelli, F. Bernardi, P. Celani, M. A. Robb, and M. Olivucci, J. Photochem. Photobiol., A 114, 109 (1998).

${ }^{22}$ A. Brodeur and S. L. Chin, J. Opt. Soc. Am. B 16, 637 (1999).

${ }^{23}$ C. W. Hillegas, J. X. Tull, D. Goswami, D. Strickland, and W. S. Warren, Opt. Lett. 19, 737 (1994); M. A. Dugan, J. X. Tull, and W. S. Warren, J. Opt. Soc. Am. B 14, 2348 (1997).

${ }^{24}$ B. J. Pearson, J. L. White, T. C. Weinacht, and P. H. Bucksbaum, Phys. Rev. A 63, 063412 (2001).

${ }^{25}$ R. S. Judson and H. Rabitz, Phys. Rev. Lett. 68, 1500 (1992).

${ }^{26}$ H.-H. Perkampus, UV-Vis Atlas of Organic Compounds (VCH, New York, 1992).

${ }^{27}$ J. L. White, B. J. Pearson, and P. H. Bucksbaum, J. Phys. B 37, L399 (2004).

${ }^{28}$ P. H. Bucksbaum, Nature (London) 396, 217 (1998).

${ }^{29}$ T. Brixner, N. H. Damrauer, B. Kiefer, and G. Gerber, J. Chem. Phys. 118, 3692 (2003); T. Brixner, B. Kiefer, and G. Gerber, Chem. Phys. 267, 241 (2001).

${ }^{30}$ S.-H. Lee, K.-H. Jung, J. H. Sung, K.-H. Hong, and C. H. Nam, J. Chem. Phys. 117, 9858 (2002).

${ }^{31}$ D. Meshulach and Y. Silberberg, Nature (London) 396, 239 (1998).

${ }^{32}$ V. V. Lozovoy, I. Pastirk, K. A. Walowicz, and M. Dantus, J. Chem. Phys. 118, 2187 (2003).

${ }^{33}$ G. Cerullo, C. J. Bardeen, Q. Wang, and C. V. Shank, Chem. Phys. Lett. 262, 362 (1996).

${ }^{34}$ J. Cao, C. J. Bardeen, and K. R. Wilson, J. Chem. Phys. 113, 1898 (2000); O. Nahmias, O. Bismuth, O. Shoshana, and S. Ruhman, J. Phys. Chem. A 109, 8246 (2005).

${ }^{35}$ D. J. Tannor and S. A. Rice, J. Chem. Phys. 83, 5013 (1985); Adv. Chem. Phys. 70, 441 (1988).

${ }^{36}$ A. M. Muller, B. Witzel, C. J. G. J. Uiterwaal, J. Wanner, and K. L. Kompa, Phys. Rev. Lett. 88, 023001 (2002).

${ }^{37}$ A. M. Weiner, D. E. Leaird, G. P. Wiederrecht, and K. A. Nelson, J. Opt. Soc. Am. B 8, 1264 (1991). 